\title{
THE CONE AXIOM IMPLIES THE HOMOTOPY AXIOM
}

\author{
RONALD J. KNILL ${ }^{1}$
}

Of the axioms for formal homology theory the homotopy axiom generally seems to have the least elegant proof. An alternative easier to prove would be the cone axiom: The cone over any space $X$ has the homology groups of its vertex. A little thought shows that for the simplicial and singular theories, at least, the cone axiom implies the homotopy axiom since one uses the former to apply either acyclic carriers of the theory of acyclic models. So it is reasonable to expect that the cone plus the remaining axioms imply the homotopy axiom. This may be seen as follows.

For a proper triad $\left(Y ; X_{1}, X_{2}\right)$ such that $Y=X_{1} \cup X_{2}[1$, p. 34] the Mayer-Vietoris sequence differs from that of $\left(Y ; X_{2}, X_{1}\right)$. In particular if

$$
\Delta: H_{*}(Y) \rightarrow H_{*}\left(X_{1} \cap X_{2}\right)
$$

is the boundary operator of the M.-V. sequence of $\left(Y ; X_{1}, X_{2}\right)$ then $-\Delta$ is the boundary operator of the M.-V. sequence of $\left(Y ; X_{2}, X_{1}\right)$.

Now suppose that

$$
\left(Y ; X_{1}, X_{2}\right)=(S X ; p X, q X),
$$

where $S X$ is $X \times[-1,1]$ with $X \times\{1\}$ identified to a point $p$ and $X \times\{-1\}$ identified to a point $q$. Denote the elements of $S X$ by their representatives $(x, t)$ in $X \times[-1,1]$; identify $x \in X$ with $(x, 0)$ $\in S X$; for $z=(x, t)$ let $-z$ denote $(x,-t)$ and for a subset $A$ of $S X$ let $-A$ be the set of all $-z$ for $z \in A$. Let

$$
p X=-q X=\{(x, t) \in S X: 0 \leqq t \leqq 1\} .
$$

If

$$
f: S X \rightarrow S X
$$

takes every $z$ into $-z$, then $f$ is a map of proper triads

$$
f:(S X ; p X, q X) \rightarrow(S X ; q X, p X)
$$

and $f$ is the identity map on $X$, so it induces the vertical homomorphisms of the diagram

Received by the editors April 5, 1965.

1 Supported in part by NSF Grant GP3989. 


$$
\begin{gathered}
H_{*}(S X) \stackrel{\Delta}{\longrightarrow} H_{*}(X) \\
f_{*} \downarrow \\
H_{*}(S X) \stackrel{-\Delta}{\longrightarrow} H_{*}(X),
\end{gathered}
$$

where $H_{*}$ is used to denote augmented homology. By the exactness of the M.-V. sequence and the cone axiom, $\Delta$ is an isomorphism so $f_{*}=-1$. On the other hand considered as a map

$$
f:(S X ; T X,-T X) \rightarrow(S X ;-T X, T X),
$$

where

$$
T X=\left\{(x, t) \in S X:-\frac{1}{2} \leqq t \leqq 1\right\},
$$

$f$ induces the vertical homomorphisms of the diagram

$$
\begin{aligned}
& H_{*}(S X) \stackrel{\Delta}{\longrightarrow} H_{*}\left(X \times\left[-\frac{1}{2}, \frac{1}{2}\right]\right) \\
& -1 \downarrow \\
& H_{*}(S X) \stackrel{-\Delta}{\longrightarrow} H_{*}\left(X \times\left[-\frac{1}{2}, \frac{1}{2}\right]\right),
\end{aligned}
$$

$f^{\prime}$ being the restriction of $f$ to $X \times\left[-\frac{1}{2}, \frac{1}{2}\right]$. Then $f_{*}^{\prime}=1$. If we let

$$
h, k: X \rightarrow X \times\left[-\frac{1}{2}, \frac{1}{2}\right]
$$

be the maps $h(x)=\left(x, \frac{1}{2}\right), k(x)=\left(x,-\frac{1}{2}\right)$, then $h=f^{\prime} k$ and $h_{*}=k_{*}$, which is the homotopy axiom.

Actually that $(S X ; p X, q X)$ is a proper triad is a little stronger than the usual Eilenberg-Steenrod excision axiom. If we replace $p X$ by $T^{\prime} X=\left\{(x, t) \mid-\frac{1}{4} \leqq t \leqq 1\right\}$ and $q X$ by $-T^{\prime} X$, the resulting triad is always proper, and the previous proof still goes through if we also replace $f$ by the map $g$ defined by letting $g(x, 1)=(x,-1), g\left(x, \frac{1}{2}\right)$ $=\left(x,-\frac{1}{2}\right), g\left(x, \frac{1}{4}\right)=\left(x, \frac{1}{4}\right)$ and $g(x, 0)=(x, 0), g$ being linear in the second variable between the given points and satisfying $g(-z)$ $=-g(z)$ for all $z$ in $S X$.

\section{REFERENCE}

1. S. Eilenberg and N. Steenrod, Algebraic topology, Princeton Univ. Press, Princeton, N. J., 1952.

TULANE UNIVERSITY 